\title{
ENTREVISTA CON OLGA LIZA SOÁIN RUMEU SOBRE PEDAGOGÍA HOSPITALARIA: TRAYECTORIAS DE DESARROLLO INTELECTUAL, CONQUISTAS PROFESIONALES Y DESAFÍOS DE FUTURO
}

\section{Aldo Ocampo González}

Director fundador del Centro de Estudios Latinoamericanos de Educación Inclusiva (CELEI), Chile

\footnotetext{
1 Chileno. Director fundador del Centro de Estudios Latinoamericanos de Educación Inclusiva (CELEI), primer centro de investigación creado en Latinoamérica y el Caribe en esta materia. www.celei.cl. Académico del Programa de Magíster en Educación Inclusiva de la Univ. Santo Tomás, La Serena, Chile. Profesor invitado en el Programa de Maestría en Educación desde la Diversidad de la Univ. de Manizales, Colombia; Profesor Máster en Creatividad, Educación y Bienestar de la Universitat de Barcelona, España; Profesor del Magíster en Gestión y Liderazgo Educacional de la Univ. La República, Chile y director de Tesis Doctorales en el Programa Oficial de Doctorado en Educación de la Univ. Internacional Sek, Chile. Doctor en Ciencias de la Educación aprobado sobresaliente por unanimidad, mención $\square$ Cum Laude $\square$ (UGR, España). Permanentemente impartes conferencias y seminarios en diversos países de Iberoamérica gracias a sus escritos. Autor de 4 libros y de más de 80 artículos científicos. Su último libro titulado: $\square$ La formación del profesorado y la comprensión epistemológica de la Educación Inclusiva $\square$. E-mail: aldo.ocampo@celei.cl ORDCID: http://orcid.org/0000-0002-6654-8269
}

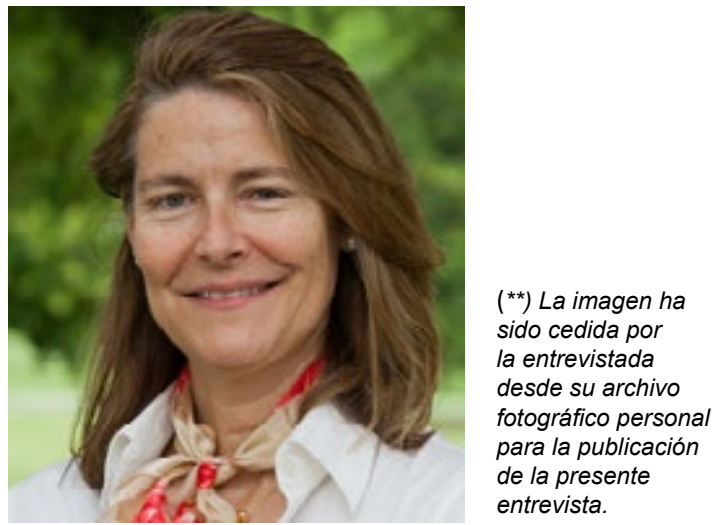

Bionota:

Doctora en Pedagogía. Profesora titular de Educación Especial y Pedagogía Hospitalaria en la Facultad de Educación y Psicología de la Universidad de Navarra. Es también Vicedecana de dicha Facultad. Desarrolla actividades de orientación familiar y atención psicopedagógica destinadas a los niños ingresados en la planta de Pediatría de la Clínica Universidad de Navarra y sus familias. Es miembro fundador de la Asociación Europea de Pedagogos Hospitalarios (HOPE) y Miembro de Honor de la Red Latinoamericana y del Caribe por el Derecho a la Educación de los niños Hospitalizados (REDLACEH). Entre sus temas de investigación figuran: la Pedagogía Hospitalaria y la atención psicoeducativa al alumno enfermo; los hermanos de personas con enfermedades y discapacidad; la formación del profesorado en la atención a la diversidad; y la Orientación Vocacional a jóvenes con dificultades de aprendizaje. 
Aldo Ocampo González (A.O.G.):

Buenas días, doctora Lizasoáin.

En primer lugar, agradezco su gentileza en la aceptación y apoyo para la realización de este ciclo de entrevistas. Para comenzar, ¿Qué es la Pedagogía Hospitalaria?, ¿Cuál es su concepción al respecto?

Olga Lizasoáin Rumeu (O.L.R.): Muchísimas gracias a ti, Aldo, por considerar mi investigación en este ciclo de entrevistas. La Pedagogía Hospitalaria $(\mathrm{PH})$ es aquella parte de la Pedagogía que se encarga de la educación de los niños y adolescentes enfermos u hospitalizados, de manera que no se retrasen en su desarrollo personal ni en sus aprendizajes, a la vez que procura atender a las necesidades afectivas y sociales generadas como consecuencia de la hospitalización y de la concreta enfermedad que padecen. Es una profesión de ayuda dependiente de la Pedagogía General, en tanto que debe regirse por las normas o principios generales de todo proceso educativo. Tiene también una gran relación de dependencia respecto de la Educación Especial, por lo que debe nutrirse de sus contenidos, así como de la didáctica general y especial, y de la orientación. Si bien durante las últimas décadas se ha identificado el término Pedagogía Hospitalaria $(\mathrm{PH})$ con el servicio educativo prestado en las aulas hospitalarias, hay que afirmar que aquella trasciende a éstas como concepto y actuación. Puede decirse así que las "aulas hospitalarias" se consideran el primer, y más sólido, escalón de la denominada Pedagogía Hospitalaria ( $\mathrm{PH})$, siendo la atención domiciliaria el segundo escalón y el colegio de origen o de referencia, donde está escolarizado el alumno, el tercero. Un cuarto escalón es la orientación y el acompañamiento familiar de los alumnos implicados.

Desde la misma hospitalización, la Pedagogía Hospitalaria $(\mathrm{PH})$ se va abriendo camino hacia las consultas ambulatorias, las unidades de neonatos y atención de adultos que, siguiendo con el símil de la escalera, se corresponderían con un quinto, sexto y sucesivos escalones. La Pedagogía Hospitalaria $(\mathrm{PH})$ se va adaptando así a los cambios de la sociedad y afronta nuevos retos educativos. Hay que tener en cuenta por lo tanto que el mundo evoluciona y que son muchos los retos que se van planteando tales como la reducción de la estancia hospitalaria, los avances de la medicina, el aumento de las enfermedades psiquiátricas en la etapa infantojuvenil, los requisitos de formación profesional, los cambios sociales y económicos o el vertiginoso desarrollo de las TIC. La Pedagogía Hospitalaria se enmarca ya en un contexto con bases legislativas, con ente propio, con agentes definidos y con una clara finalidad. Ha costado mucho llegar aquí, y se trata de preservar lo ya alcanzado, sin cuestionarlo gratuitamente, ni mucho menos tergiversarlo, si no es para construir y avanzar sobre los principios establecidos. No se vaya a correr el riesgo de que con el deseo implícito de querer "ir más allá" a toda costa, se hagan tambalear las bases que con tanto esfuerzo se encuentran ya asentadas.

A.O.G.: En relación a lo anteriormente comentado, ¿qué desafíos enfrenta la pedagogía hospitalaria como campo de conocimiento en este nuevo siglo?

O.L.R.: Creo que ya he apuntado alguna respuesta a esta pregunta en el punto anterior. No obstante, profundizaré algo más diciendo que la Pedagogía Hospitalaria (PH) está enmarcada por el hecho incontestable de la enfermedad y por el entorno hospitalario donde se lleva principalmente a cabo. El hospital para un niño, para un adolescente, es un lugar que está fuera de lugar, que no tiene lógica. Es un lugar donde se plantean las preguntas: ¿por qué yo? ¿por qué a mí? ¿qué me va a pasar? Preguntas para las que es muy difícil encontrar 
una respuesta. El hospital para un niño es un lugar donde no se come la misma comida, ni se duerme a la misma hora, no se obedece a las mismas personas, ni tampoco se siguen los mismos parámetros de vida. $\mathrm{Y}$ en este contexto, fuera de contexto, surge la figura del profesor hospitalario, del profesor de atención domiciliaria, del profesional de la educación o del pedagogo, algo familiar, algo cercano, con reglas conocidas, y surgen preguntas como: ¿por qué estudiar si estoy enfermo? ¿por qué un colegio en el hospital? Personas cercanas al entorno de un alumno en situación de enfermedad deben reflexionar sobre estos interrogantes para encontrar respuestas que den sentido a las acciones psicoeducativas $\mathrm{y}$, por supuesto, a la Pedagogía Hospitalaria (PH). La educación trata de paliar necesidades (de saber, afectivas, espirituales...) y de descubrir posibilidades. En función de las necesidades y posibilidades, se adapta la intervención psicoeducativa, se individualiza, se singulariza. Para esto es importante conocer al educando, incluyendo a su familia. Educar al niño enfermo significa además enseñarle desde su enfermedad, no a pesar de su enfermedad. La legislación española, así como la mayoría de las políticas educativas del resto de los países europeos y latinoamericanos, han incluido la Pedagogía Hospitalaria $(\mathrm{PH})$ dentro del ámbito de la atención a la diversidad, como educación de alumnos con necesidades específicas de apoyo educativo por causa de una enfermedad. Como es evidente que cuando un alumno está enfermo no va a poder asistir al centro educativo con normalidad, se crean tal y como he referido líneas atrás, las aulas hospitalarias $\mathrm{y}$, posteriormente, se establece la atención educativa a domicilio, con el fin de que esos alumnos no rompan su ritmo escolar, no se retrasen en su aprendizaje ni en su desarrollo personal, a la vez que se procura atender a las necesidades psicológicas y sociales generadas por la enfermedad y la hospitalización.
A.O.G.: Usted es reconocida como una investigadora fundadora de la Pedagogía Hospitalaria en España y Latinoamérica. De acuerdo con esto y considerando su amplia trayectoria, ¿podría ofrecernos una síntesis sobre el desarrollo histórico y/o conceptual de la Pedagogía Hospitalaria, resaltando los diversos objetos de conocimiento que han sido formados en su desarrollo?

O.L.R.: A pesar de su aparente juventud, la Pedagogía Hospitalaria $(\mathrm{PH})$ tiene ya una larga andadura. La actividad pedagógica como complemento de la acción médica en los hospitales ha tomado carta de naturaleza desde hace años en muy diversos países europeos, norteamericanos $y$, más recientemente, de América latina. En décadas pasadas los departamentos infantiles se caracterizaban por un ambiente rígido donde no había lugar para la educación de los niños. Desde que René Spitz describiera, en 1945, el síndrome de hospitalismo, sobre las consecuencias negativas que el ingreso en un centro médico tenía sobre el desarrollo infantil, podemos decir que la Pedagogía Hospitalaria $(\mathrm{PH})$ ha ido poco a poco implementándose y evolucionando impulsada, fundamentalmente, por los siguientes factores: a) profesionales de la salud y de la educación, b) asociaciones de padres de niños enfermos, c) voluntariados, d) las administraciones educativas y e) el desarrollo de la conciencia social. Junto a estos, otro elemento de enorme importancia son las Cartas de Derechos relacionadas con los pacientes pediátricos, entre las que destaco tres: a) la Carta Europea de los Derechos del Niño Hospitalizado, aprobada por el Parlamento Europeo en 1986, b) la Carta Europea sobre el Derecho a la Atención Educativa de los Niños y Adolescentes Enfermos, aprobada en la Asamblea General de la asociación HOPE (Hospital Organisation of Pedagogues in Europe) en el año 2000, c) la Declaración de los Derechos del Niño, Niña o Joven Hospitalizado o en Tratamiento 
de América Latina y el Caribe en el Ámbito de la Educación, validada por el Parlamento Latinoamericano en noviembre de 2013. En España, podemos decir que, desde 1982, la Ley sobre la Integración Social de los Minusválidos (LISMI), recogió por vez primera la obligatoriedad de los hospitales de contar con una sección pedagógica para prevenir y evitar la marginación del proceso educativo de los alumnos en edad escolar ingresados. Gracias a esta normativa se fueron creando las aulas hospitalarias o unidades escolares de apoyo en instituciones hospitalarias, tal y como se conocen en la actualidad. Estas aulas están atendidas por profesores de las etapas de educación infantil, primaria o secundaria, que trabajan bajo las mismas condiciones respecto a horarios $\mathrm{y}$ calendario laboral que un profesor de escuela del sistema educativo regular. Junto a esto, y debido a que el número medio de días de ingreso va en clara disminución, se va desarrollando un amplio campo de acción para la pedagogía hospitalaria que es el Servicio de Atención Educativa a Domicilio (SAED). El objetivo es que el alumno enfermo reciba esta atención durante el período de convalecencia en su casa, mientras no pueda acudir al colegio en el que está escolarizado. Esta atención domiciliaria en sus inicios corrió a cargo de organizaciones no gubernamentales y de voluntarios, pero, poco a poco, tras su progresiva implementación y justificada necesidad, pasó también a legislarse (en España se recoge una primera legislación en el Real Decreto, de 1996, de ordenación de las acciones dirigidas a la compensación de desigualdades en educación).

Puede darse también el caso de alumnos que sin estar hospitalizados, con una enfermedad que les impide ir al colegio regularmente pero que sí están en condiciones de salir de casa, acudan diariamente al aula hospitalaria como un sustituto de la atención domiciliaria. Como se va viendo, la Pedagogía Hospitalaria $(\mathrm{PH})$ es acorde con los principios que rigen la atención a la diversidad y la educación de los alumnos con necesidades específicas de apoyo educativo. Cada país, comunidad, distrito o región los va adaptando en función de sus propias características, políticas socioeducativas y recursos disponibles.

Quiero destacar que la Pedagogía Hospitalaria en Latinoamérica ha sufrido un desarrollo espectacularenlasúltimasdécadas.Asociaciones como "Carolina Labra Riquelme" en Chile, "El aula de los sueños" en Venezuela, "Aprendo contigo" en Perú, "Semillas del corazón" en Argentina, "Sigamos aprendiendo en el hospital" en México, instituciones como Fundación Telefónica y otras tantas han impulsado la atención educativa a los niños enfermos y hospitalizados, creando las bases de actuación y la concienciación necesarias para que los respectivos Ministerios de Educación vayan implicándose de manera directa y asumiendo sus responsabilidades. Mención especial merece REDLACEH, la Red Latinoamericana y del Caribe por el Derecho a la Educación de Niños y Jóvenes Hospitalizados o en Tratamiento. Uno de los retos más apremiantes que tiene la educación en Latinoamérica es el fomento de la igualdad de oportunidades y la lucha contra la exclusión social y la discriminación, en el caso que nos ocupa, poniendo el énfasis en el colectivo de alumnos que se encuentran en situación de vulnerabilidad por motivo de una enfermedad. Así, en la mayoría de los países se cuenta ya con el marco legal propicio, o al menos con diversas declaraciones de intenciones, que facilitan la movilización que exige este afrontamiento.

A.O.G.: ¿Cuáles deberían ser los ejes clave para pensar la Pedagogía Hospitalaria como proyecto ético y pedagógico?

O.L.R.: Para sustentar el proyecto ético y pedagógico de la Pedagogía Hospitalaria $(\mathrm{PH})$ emplearé las siguientes palabras: "El que no 
entienda que una persona ingresada en un hospital tiene unas necesidades de atención que van más allá de lo 'medicofísico', que un niño en el hospital tiene que seguir con las actividades que le son propias como estudiar, jugar, hablar, reírse, estar con otros niños; el que no entienda que un niño con una enfermedad grave tiene derecho a seguir aprendiendo, interesándose por las cosas, realizando actividades, jugando; el que no entienda que esos padres, con un hijo enfermo crónico, tienen necesidad de orientación, [...] tiene, sencillamente, una concepción errónea o parcial de lo que es la vida (Lizasoáin, 2000). De las diferentes experiencias e investigaciones en el ámbito de la Pedagogía Hospitalaria $(\mathrm{PH})$ puede afirmarse que la atención educativa al niño enfermo y hospitalizado contribuye a su estabilidad emocional y a una más pronta recuperación. La enseñanza escolar en los hospitales humaniza la estancia del niño y sirve de prevención ante ciertos efectos negativos que el tratamiento médico y el propio hospital pueden originar. Aunque el énfasis principal se centra en la enseñanza escolar, no se trata tan solo de impartir una serie de materias y contenidos al alumno enfermo y hospitalizado. Los niños precisan, para lograr una adecuada adaptación, de una gran variedad de actividades recreativas y de elaciones personales. Junto a esto no puede dejarse de lado a la familia de los pacientes, es decir, a los padres y hermanos de los niños hospitalizados. Para profundizar algo más en estas ideas, quiero exponer algunas más sobre la justificación de la Pedagogía Hospitalaria (PH). Así, la finalidad de la Pedagogía Hospitalaria es la misma que la de toda la educación, es decir, el desarrollo integral de la persona, el desarrollo máximo de todas sus capacidades incluso en la situación de enfermedad y hospitalización. El desglose de esta finalidad general dará lugar a una amplia relación de objetivos fundamentales que conseguir mediante la actividad pedagógica en el paciente pediátrico, entre los que se citan los siguientes: a) mejorar su calidad de vida y la de su familia, b) favorecer la adaptación a la hospitalización y a la situación de enfermedad, c) disminuir la ansiedad y los miedos, e) atender a la formación del carácter y de la voluntad, f) fomentar la actividad, procurando que el niño ocupe provechosamente su tiempo libre, g) cultivar la natural alegría infantil y h) Ilevar a cabo una orientación personal y profesional.

La Pedagogía Hospitalaria $(\mathrm{PH})$ se propone luchar contra la pérdida del hábito intelectual, apelar a la importancia del esfuerzo, fomentar la conciencia de la responsabilidad, reducir las lagunas de aprendizaje y el retraso escolar, prevenir las alteraciones emocionales, luchar contra el síndrome del hospitalismo, provocar una despolarización respecto de la enfermedad y evitar el aburrimiento. La Pedagogía Hospitalaria $(\mathrm{PH})$ se centra en la parte sana de la persona y envía un mensaje de recuperación, es una actividad conocida, propia de un niño o un adolescente, añade interés a su día, le proporciona seguridad y confianza. La Pedagogía Hospitalaria (PH) favorece así la readaptación tras la enfermedad y la hospitalización, facilitando el camino para la vuelta al colegio. Y sabemos muy bien que el listado podría continuar.

A.O.G.: ¿Qué sugerencias y/o recomendaciones podría ofrecer al campo de la formación de maestros en materia de Pedagogía Hospitalaria?

O.L.R.: Comenzaré respondiendo a esta cuestión enfatizando los cuatro roles básicos que todo profesional de la educación que trabaja en Pedagogía Hospitalaria $(\mathrm{PH})$ debería asumir para llevar a cabo una intervención educativa de calidad, tanto con el alumno enfermo como con su familia.

- La colaboración interdisciplinar.

- La evaluación psicosocial del alumno.

- La aplicación de estrategias de intervención 
psicoeducativa.

- La investigación y la difusión de la actividad pedagógica

$Y$ me quiero centrar en este último pues se dice que lo que no se publica no se difunde, lo que no se difunde no se lee, lo que no se lee no se conoce y lo que no se conoce, es como si no existiera. La investigación es nuclear para compartir y dar a conocer el trabajo que se hace desde la Pedagogía Hospitalaria $(\mathrm{PH})$ con el fin de que no se quede solo en las actividades de un determinado profesor, sino que sirva de ejemplo, de modos de hacer y de buenas praxis para otros profesionales. A su vez esto es básico para hacer que la ciencia y el conocimiento avancen, así como para contribuir a la formación de los futuros profesionales de la Pedagogía Hospitalaria $(\mathrm{PH})$ y de los que ya están en activo. Hay demanda de información y de intercambio de experiencias en este ámbito. Junto a esto, existe la necesidad de una formación específica del profesorado para abordar la educación de los alumnos enfermos, tanto cuando están hospitalizados o convalecientes en el domicilio, como cuando se trata de su inclusión en la escuela regular.

Los temas de formación que más demandan los profesionales están relacionados con los siguientes aspectos: a) conocimiento actualizado de las posibles enfermedades infantiles, b) de los tratamientos médicos y de sus efectos; c) pautas para la orientación familiar; d) estrategias para adaptar el currículo y para la utilización de recursos informáticos y audiovisuales en las aulas hospitalarias; e) apoyos para entablar relaciones con el colegio de origen; y f) competencias en los dominios de la escucha y del acompañamiento de los enfermos. Una reflexión acerca del concepto de muerte para el niño se reclama también como parte de la formación profesional a la que se está haciendo referencia. Por último, aparece la necesidad de trabajar estrategias de cooperación educativa y el intercambio de experiencias para generar trabajo colaborativo entre los profesionales. El objetivo es abordar las relaciones del profesorado del aula hospitalaria con el profesorado de domiciliaria, con el de los tutores del colegio ordinario, con el profesorado de apoyo a las necesidades especiales, con los departamentos de orientación y con las instituciones educativas.

Los cambios sociales de las últimas décadas depositan muchas responsabilidades en los profesionales de la educación. La opción de una Educación Inclusiva, de una educación de calidad para todos, demanda un profesorado comprometido, bien formado y competente, capaz de hacer que sus alumnos aprendan al máximo, independientemente de las características que presenten.

Todas estas premisas e ideas aquí apuntadas deben guiar el establecimiento de las bases para una adecuada formación en Pedagogía Hospitalaria $(\mathrm{PH})$, necesaria para llevar a cabo una educación de calidad, reclamada desde la dignidad y la defensa de los derechos del colectivo de niños enfermos y hospitalizados. Para terminar quiero insistir, una vez más, en un punto esencial: la formación no puede concebirse más que como formación continua. No basta con estar preparados, con obtener un reconocimiento oficial que nos permita un día trabajar junto a los niños enfermos. Un buen trabajo con un estudiante en situación de enfermedad exige y merece una formación permanente. A cada uno le compete tomar sus responsabilidades. El ejercicio y el entrenamiento podrían ser suficientes para mantener las competencias esenciales si la Pedagogía Hospitalaria fuera «un mundo estable». Ahora bien, la profesión se ejerce dentro de contextos inéditos, ante pacientes que cambian, con referencias a programas que se replantean constantemente, apoyados por nuevos conocimientos, nuevos enfoques, 
nuevos paradigmas. De ahí la necesidad de una formación continua, resaltando el hecho de que los recursos cognitivos, movilizados por las competencias, deben ponerse al día, para adaptarse a unas condiciones de trabajo en constante evolución.

A.O.G.: Altamente significativo el escenario que nos ofrece sobre el desarrollo históricoconceptual y sociopolítico de la Pedagogía Hospitalaria como campo de investigación y desarrollo profesional.

A nombre del Centro de Estudios Latinoamericanos de Educación Inclusiva (CELEI), agradezco la oportunidad de dialogar con usted acerca de tales temáticas.

O.L.R.: A ti, gracias por esta invitación.

\section{Referencias}

Lizasoáin, O. (2000). Educando al niño enfermo: perspectivas de la Pedagogía Hospitalaria. Pamplona: Eunate. 